\title{
Effects of glatiramer acetate on fatigue and days of absence from work in first-time treated relapsing-remitting multiple sclerosis Tjalf Ziemssen*1, Josef Hoffman², Rainer Apfel ${ }^{2}$ and Simone Kern ${ }^{1}$
}

\author{
Address: ${ }^{1}$ MS Center, Neurological University Clinic, Technical University of Dresden, Dresden, Germany and ${ }^{2}$ TEVA Germany, Mörfelden, \\ Germany \\ Email: Tjalf Ziemssen* - Tjalf.Ziemssen@uniklinikum-dresden.de; Josef Hoffman - Josef.Hoffmann@teva.de; \\ Rainer Apfel - Rainer.Apfel@teva.de; Simone Kern - Simone.Kern@uniklinikum-dresden.de \\ * Corresponding author
}

Published: 5 September 2008

Health and Quality of Life Outcomes 2008, 6:67 doi:10.1 186/1477-7525-6-67

This article is available from: http://www.hqlo.com/content/6/1/67

(C) 2008 Ziemssen et al; licensee BioMed Central Ltd.

This is an Open Access article distributed under the terms of the Creative Commons Attribution License (http://creativecommons.org/licenses/by/2.0), which permits unrestricted use, distribution, and reproduction in any medium, provided the original work is properly cited.
Received: 16 May 2008

Accepted: 5 September 2008

\begin{abstract}
Objectives: Treatment of multiple sclerosis patients with glatiramer acetate has been demonstrated a beneficial effect on disease activity. The objective of this prospective naturalistic study was to evaluate the impact of glatiramer acetate on fatigue and work absenteeism.

Methods: 29 I treatment-naïve patients with relapsing remitting multiple sclerosis were included and treated with glatiramer acetate for twelve months. Relapse rates, disability, fatigue symptoms, days of absence from work and adverse events were monitored. Fatigue was measured with the MFIS scale and with a visual analogue scale.

Results: Total MFIS scores decreased by $7.6 \pm 16.4$ from 34.6 to $27.0(p \leq 0.00 I)$. Significant reductions were observed on all three subscales of the MFIS. Fatigue symptoms, assessed using a visual analogue scale, decreased by $1.04 \pm 2.88 \mathrm{~cm}$ from $4.47 \mathrm{~cm}$ to $3.43 \mathrm{~cm}(p \leq 0.00 \mathrm{I})$. The proportion of patients absent from work at least once was reduced by a factor of two from $65.1 \%$ to $30.1 \%(p \leq 0.00 \mathrm{I})$. Tolerance to treatment was rated as very good or good in $78.3 \%$ of patients. Adverse effects, most frequently local injection site reactions, were reported in $15.1 \%$ of patients.

Conclusion: Treatment with glatiramer acetate was associated with a significant improvement in fatigue symptoms and a marked reduction in absence from work. Treatment was well-tolerated. Such benefits are of relevance to overall patient well-being.
\end{abstract}

\section{Introduction}

Fatigue is a common symptom of multiple sclerosis [1-5], reported by around three-quarters of affected patients [3], and considered one of the most distressing symptoms of disease by over half [2]. Many patients experience debilitating fatigue every day [2]. In multiple sclerosis, fatigue has a major detrimental impact on quality of life [6-8], is frequently associated with depression $[9,10]$ and is a leading cause of absence from work or impaired work per- formance $[6,11,12]$. The pathophysiology of fatigue in multiple sclerosis is poorly understood, but is likely to be multifactorial [13-16]

Treatment of fatigue in multiple sclerosis is thus a major challenge, which cannot be adequately achieved at the present time. Both non-pharmacological and pharmacological interventions have been proposed for the management of fatigue in multiple sclerosis patients $[15,17]$, 
although the benefits of drugs such as modafenil and amantadine have not been demonstrated unequivocally [18-20].

Immunomodulatory treatments for relapsing-remitting multiple sclerosis, namely glatiramer acetate and the $\beta$ interferons, provide a marked reduction in relapse rates and in MRI markers of disease activity [21]. It is therefore of interest to explore whether such treatments might influence fatigue symptoms as well. A retrospective chart review of 218 Canadian patients receiving an immunomodulatory treatment during the late 1990s revealed that fatigue improved over the six months following treatment initiation [22]. Of particular interest was the observation that a significantly higher proportion of glatiramer acetate treated patients than $\beta$-interferon-treated patients improved by at least one standard deviation of the Fatigue Impact Scale (FIS).

In order to investigate further the potential impact of immunomodulatory treatment on fatigue in multiple sclerosis, we initiated a prospective, observational, noninterventional study to monitor fatigue in treatment-naive RRMS patients initiating therapy with glatiramer acetate under conditions of daily practice. The primary objective of study was to determine the impact of initiating treatment with glatiramer acetate on fatigue and absenteeism. Secondary objectives were to evaluate the effect of treatment on clinical and MRI outcomes and to determine the tolerability of treatment.

\section{Methods}

This study was a prospective, observational, non-interventional study of patients with relapsing remitting multiple sclerosis treated with glatiramer acetate conducted in Germany. 130 ambulatory and hospital neurologists participated in the study. The study was performed between November 2002 and October 2004.

\section{Patients}

The study included patients with a diagnosis of relapsingremitting multiple sclerosis by the McDonald criteria [23] who had not previously been treated with an immunomodulatory treatment and in whom the investigator had decided to initiate therapy with glatiramer acetate. Patients were followed for twelve months following treatment initiation.

\section{Clinical assessment}

Patients were evaluated at inclusion and after 3, 6, 9 and 12 months of treatment. At each visit, patients underwent a full neurological assessment, any relapses occurring since the previous visit were ascertained and disability assessed with the Expanded Disability Status Scale (EDSS) [24]. Fatigue was assessed by the patient using a visual analogue scale scored from 0 (no fatigue) to 10 (maximum possible fatigue) and with the Modified Fatigue Impact Scale (MFIS) [25] in its validated German translation. This is a 21 -item questionnaire which yields a total score ranging from 0 (no impact of fatigue) to 84 points (maximum impact of fatigue), as well as three subscales representing the physical (score range 0 to 36), cognitive (score range 0 to 40 ) and psychosocial (score range 0 to 8 ) dimensions of fatigue.

Patients were questioned about any time spent off work due to their multiple sclerosis. Due to the study protocol, the reasons for work absentism (relapse, fatigue) could not be differentiated. Any adverse events occurring since the previous visit were recorded.

\section{Statistical analysis}

Number of work days lost and fatigue scores over the course of the study were evaluated with the Wilcoxon rank test. All comparisons were two-tailed and a $p$ value of $<$ 0.05 was taken as being statistically significant.

\section{Ethics}

This study was conducted according to the Declaration of Helsinki (Hong Kong Amendment) and pertinent national legal and regulatory requirements. Each patient provided written, informed consent and was free to withdraw from the study at any time for any reason without consequences on the care provided.

\section{Results}

\section{Study sample}

A total of 338 patients were included in the study. Of these, 53 were excluded from the analysis due to a protocol violation (24 patients treated previously with an immunomodulatory therapy and 29 patients for whom certain data were recorded retrospectively) and 47 failed to provide complete questionnaire data. The study population thus consisted of 291 subjects $(86.1 \%$ of included patients).

The baseline demographic and disease variables of the study subjects are presented in Table 1 . At inclusion, their median age was 36.9 years and $74.9 \%$ were female. The median time since diagnosis was 4.31 years. In the year preceding inclusion, patients had experienced a mean of 1.71 relapses (retrospectively assessed) and their mean EDSS score at inclusion was 2.58. Forty patients (13.7\%) discontinued treatment during the course of the study, principally due to the occurrence of an adverse event (sixteen patients).

\section{Clinical outcome}

Clinical outcome at the end of the study are presented in Table 2. Information on relapses was missing for 24 
Table I: Demographic and clinical characteristics of patients at inclusion

\begin{tabular}{lc}
\hline & Population $(\mathrm{N}=29 \mathrm{I})$ \\
\hline Age (mean \pm SD; years) & $36.9 \pm 9.3$ \\
\hline Gender & \\
Women & $218(74.9 \%)$ \\
Men & $67(23.0 \%)$ \\
Missing data & $6(2.1 \%)$ \\
& \\
Time since diagnosis (mean \pm SD; years) & $4.31 \pm 5.47$ \\
& \\
ARR since diagnosis (mean \pm SD) & $3.82 \pm 3.54$ \\
No relapses & $14(4.8 \%)$ \\
Up to 2 relapses & $111(38.1 \%)$ \\
$3-5$ relapses & $100(34.4 \%)$ \\
More than 5 relapses & $57(19.6 \%)$ \\
& \\
ARR within previous 12 months (mean \pm SD) & $1.71 \pm 0.88$ \\
EDSS at treatment initiation (mean \pm SD) & $2.58 \pm 1.44$ \\
EDSS 0-2 & $127(43.6 \%)$ \\
EDSS 3-5 & $121(41.6 \%)$ \\
EDSS 6-7 & $16(5.5 \%)$ \\
Missing data & $27(9.3 \%)$ \\
\hline
\end{tabular}

ARR: annualised relapse rate: EDSS: Expanded Disability Status Scale; SD: standard deviation.

patients. Of the remaining 267 patients, 61 (22.8\%) experienced a single relapse during the twelve-month study period and 23 patients $(8.6 \%)$ more than one relapse. The mean annual relapse rate during the year of treatment with glatiramer acetate was 0.46 . The mean EDSS score at the end of the study was 2.45 , representing a mean decrease from baseline of 0.55 points. The change in EDSS score between baseline and twelve months was statistically significant $(p<0.05$; Wilcoxon rank test). A sustained reduction in EDSS score of $>1$ point was observed

Table 2: Clinical outcome

\begin{tabular}{lc}
\hline & Population $(\mathrm{N}=29 \mathrm{I})$ \\
\hline $\begin{array}{l}\text { Relapses during study }(n=267) \\
\text { No relapse }\end{array}$ & $180(67.4 \%)$ \\
I relapse & $61(22.8 \%)$ \\
2 relapses & $12(4.5 \%)$ \\
3 relapses & $8(3.0 \%)$ \\
$4-5$ relapses & $3(1.1 \%)$ \\
& \\
Mean EDSS scores $(n=235)$ & \\
Baseline & $2.58 \pm 1.45$ \\
Study end & $2.45 \pm 1.52$ \\
Change from baseline & $-0.13 \pm 0.73 *$
\end{tabular}

Data are presented as number of patients (\%) for relapses and as mean \pm SD for Expanded Disability Status Scale (EDSS) scores. The asterisk indicates a significant change from baseline $(p<0.05$; Wilcoxon signed rank test). in fifteen patients (5.2\%) and a sustained increase of $>1$ point in three patients $(1 \%)$.

\section{Fatigue}

Overall, 220 patients provided exploitable data from the MFIS questionnaire at both inclusion and study end. Measures were compared between the three-month period before inclusion and the last three months of the treatment period. Significant decreases were observed in the total score as well as in all three dimension scores of the MFIS (Table 3). Similarly, the VAS rating of fatigue was reduced by around one quarter following initiation of treatment with glatiramer acetate (Table 3 ), between baseline and study end

\section{Work absenteeism}

The number of days missed from work due to multiple sclerosis was evaluated in the patients who were in employment $(72.9 \%$ of the study population). In the three month period preceding inclusion, 138 patients $(65.1 \%)$ had taken at least one day off work (Tables 4 and $5)$. This number decreased to 64 patients $(30.1 \%)$ in the year following initiation of treatment with glatiramer acetate. The number of days lost was significantly lower in the second year ( $p \leq 0.001$; Wilcoxon rank test).

\section{Safety}

Safety was assessed in all 338 included patients. Overall, 51 patients $(15.1 \%)$ experienced at least one adverse event during the treatment period. These were most frequently injection site reactions or symptoms of a systemic immediate post-injection reaction such as dyspnoea or tachycardia. No single event was reported in more than ten patients. The immediate post-injection reaction was classified as serious in one patient.

\section{Discussion}

In this study, immunomodulatory treatment of relapsingremitting multiple sclerosis with glatiramer acetate was associated with a reduction in subjective perceptions of fatigue and with the numbers of days taken off work due to illness. We observed a reduction of approximately onequarter in both MFIS scores and in a VAS measure of fatigue. These findings are consistent with an earlier retrospective study, which also reported an improvement in fatigue measured with the FIS following initiation of glatiramer acetate treatment in $24.8 \%$ of patients [22]. The two studies cannot, however, be directly compared due differences in methodology.

The amelioration observed following treatment with glatiramer acetate may be a non-specific consequence of improved overall disease status in treated patients or alternatively result from a specific action of the medication on the pathophysiology of multiple sclerosis fatigue. For 
Table 3: Fatigue ratings.

\begin{tabular}{lcccc}
\hline & Baseline & On treatment & Mean Change & $p$ \\
\hline MFIS Total score $(n=220)$ & $34.6 \pm 18.7$ & $27.0 \pm 18.6$ & $-7.6 \pm 16.4$ & $p \leq 0.00 \mathrm{I}$ \\
Physical dimension score & $17.6 \pm 9.1$ & $13.5 \pm 9.0$ & $-4.1 \pm 8.1$ & $p \leq 0.00 \mathrm{I}$ \\
Cognitive dimension score & $13.9 \pm 9.2$ & $11.2 \pm 8.6$ & $2.7 \pm 8.0$ & $p \leq 0.00 \mathrm{I}$ \\
Psycho-social dimension score & $3.1 \pm 2.1$ & $2.4 \pm 2.0$ & $-0.7 \pm 2.0$ & $p \leq 0.00 \mathrm{I}$ \\
\hline VAS score $(n=198)$ & $4.47 \pm 2.53$ & $3.43 \pm 2.55$ & $-1.04 \pm 2.88$ & $p \leq 0.00 \mathrm{I}$ \\
\hline
\end{tabular}

Fatigue over three months was measured with the Modified Fatigue Impact Scale (MFIS) and with a visual analogue scale (VAS). Data are presented as mean \pm SD for those patients providing exploitable data both at inclusion and at study end. Probabilities were calculated with the Wilcoxon rank test.

example, it has been suggested that fatigue may be aggravated by the production of high levels of pro-inflammatory cytokines $[26,27]$. The ability of glatiramer acetate to attenuate the secretion and activity of these cytokines within the central nervous system $[28,29]$ may provide such a specific mechanism. Others have proposed, on the basis of magnetic resonance spectroscopy (MRS) findings, that fatigue may be associated with axonal injury in the cortex rather than inflammatory white matter lesions per se [30]. In a recent trial, Tedeschi et al. could demonstrate that among MS patients with low disability those with high-fatigue show higher white and gray matter atrophy and higher lesion load. They suggest that in MS, independent of disability, white and gray matter atrophy is a risk factor to have fatigue [31]. In additon, a recent trial of Rocca et al. using functional imaging in MS patients with fatigue and interferon beta-1a treatment pointed out that an abnormal recruitment of the fronto-thalamic circuitry is associated with interferon-induced fatigue in MS patients [32]. In contrast to the interferon's, the specific action of glatiramer acetate to improve MRS markers of axonal injury in multiple sclerosis might contribute to a reduction in fatigue $[33,34]$.

We also observed a dramatic reduction of over fifty percent in the number of patients who needed to take time off work due to their multiple sclerosis. This is consistent with findings from an American study, which also

Table 4: Number of days missing from work in the previous year at baseline and one year after start of treatment.

\begin{tabular}{lcccc}
\hline & \multicolumn{2}{c}{ Baseline } & \multicolumn{2}{c}{ After 12 Months } \\
& $\mathrm{N}$ & $\%$ & $\mathrm{~N}$ & $\%$ \\
\hline No & 76 & $26.1 \%$ & 148 & $50.9 \%$ \\
$\leq 5$ days & 26 & $8.9 \%$ & 27 & $9.3 \%$ \\
$6-10$ days & 39 & $13.4 \%$ & 14 & $4.8 \%$ \\
II-20 days & 32 & $11.0 \%$ & 8 & $2.8 \%$ \\
$>20$ days & 50 & $17.2 \%$ & 18 & $6.2 \%$ \\
Not in employment & 68 & $23.4 \%$ & 62 & $21.3 \%$ \\
Missing information & 0 & $0 \%$ & 14 & $4.8 \%$ \\
\hline
\end{tabular}

reported a marked decrease in days off work in patients treated with glatiramer acetate [35], but less so with betainterferons. This is an important functional effect of treatment since the ability to hold down a job satisfactorily is critical for self-esteem and because, in certain countries such as the USA, remaining in full-time employment is an important determinant of obtaining insurance for reimbursement of treatment costs.

Again, the impact of glatiramer acetate on time off work may be an indirect consequence of reduced relapse frequency, although the data from the US study showing a differential effect on time off work between glatiramer acetate and $\beta$-interferons would argue against this. Alternatively, the observed effect may be secondary to a reduction in fatigue, which has been identified in other studies to be a major reason why patients with multiple sclerosis need to take time off work $[11,12]$. Finally, it should be noted that the low incidence of debilitating side-effects reported with glatiramer acetate [36] means that patients are unlikely to need to take time off work due to treatment side-effects.

The strength of this study include the naturalistic design, which means that the findings can probably be generalised to standard care, at least in Europe, with confidence, the prospective nature of the data collection and the relatively large numbers of patients evaluated. Limitations include the absence of a comparator group against which the magnitude of the observed treatment effects could be assessed, and data collection during physician consultations rather than with patients' diaries, which may have introduced some degree of anamnestic error into the findings. The absence of a control group might overestimate the improvement in fatigue symptoms. As a placebo group is probably not ethical it will be further of interest to compare prospectively the benefit on fatigue in a group of naive MS patients treated with GA vs. a group treated with IFN-beta in a next study. 
Table 5: Development of the different groups at baseline (No work absentism, less than 5 days,...) one year after start of treatment with glatiramer acetate using the same categories (No work absentism, less than 5 days,...).

\begin{tabular}{|c|c|c|c|c|c|c|c|c|c|c|c|c|}
\hline \multirow{2}{*}{$\begin{array}{l}\text { Baseline } \\
\text { After } 12 \text { Months }\end{array}$} & \multicolumn{2}{|c|}{ No work absentism } & \multicolumn{2}{|c|}{$\leq 5$ days absent } & \multicolumn{2}{|c|}{ 6-10 days absent } & \multicolumn{2}{|c|}{ II-20 days absent } & \multicolumn{2}{|c|}{$>20$ days absent } & \multicolumn{2}{|c|}{ Not in employment } \\
\hline & $\mathrm{N}$ & $\%$ & $N$ & $\%$ & $\mathrm{~N}$ & $\%$ & $\mathrm{~N}$ & $\%$ & $N$ & $\%$ & $\mathrm{~N}$ & $\%$ \\
\hline No work absentism & 59 & $21.3 \%$ & 15 & $5.4 \%$ & 20 & $7.2 \%$ & 23 & $8.3 \%$ & 22 & $7.9 \%$ & 9 & $3.3 \%$ \\
\hline$\leq 5$ days absent & 6 & $2.2 \%$ & 8 & $2.9 \%$ & 8 & $2.9 \%$ & I & $0.4 \%$ & 4 & $1.4 \%$ & 0 & $0 \%$ \\
\hline $6-10$ days absent & 4 & $1.4 \%$ & I & $0.4 \%$ & 3 & $1.1 \%$ & 2 & $0.7 \%$ & 4 & $1.4 \%$ & 0 & $0 \%$ \\
\hline II-20 days absent & 2 & $0.7 \%$ & 2 & $0.7 \%$ & I & $0.4 \%$ & 2 & $0.7 \%$ & I & $0.4 \%$ & 0 & $0 \%$ \\
\hline$>20$ days absent & 0 & $0 \%$ & 0 & $0 \%$ & 3 & $1.1 \%$ & I & $0.4 \%$ & 13 & $4.7 \%$ & I & $0.4 \%$ \\
\hline Not in employment & 3 & $1.1 \%$ & 0 & $0 \%$ & 2 & $0.7 \%$ & 1 & $0.4 \%$ & 4 & $1.4 \%$ & 52 & $18.8 \%$ \\
\hline
\end{tabular}

\section{Conclusion}

In conclusion, this non-interventional prospective study demonstrated that treatment with glatiramer acetate was associated with a reduction in patient-reported fatigue ratings and in days missing from work, concomitant with an improvement in clinical manifestations of disease activity. These functional outcomes are of critical importance for overall patient well-being.

\section{Competing interests}

$\mathrm{JH}$ and RA are employed by TEVA Germany. TZ has received honoraria and financial compensation by Bayer Healthcare, Biogen Idec, Merck Serono, Pfizer, SanofiAventis and Teva. SK has received honoraria and financial compensation by Bayer Healthcare, Biogen Idec, SanofiAventis and Teva. Research Projects of TZ and SK were funded by the Roland-Ernst-Foundation, Robert-PflegerFoundation, Sanofi-Aventis/TEVA and Bayer Healthcare. In the MS center Dresden, clinical studies are performed for Bayer Healthcare, Biogen Idec, BioMS, Genzyme, Glaxo Smith Kline, Sanofi-Aventis and Teva.

\section{Authors' contributions}

$\mathrm{RA}, \mathrm{JH}$ and TZ were responsible for the conception of the study. TZ drafted the article. All authors contributed to the interpretation of the results and revising the article for important intellectual content. All authors read and approved the final manuscript.

\section{Acknowledgements}

This was an investigator-driven, only observational study supported by an unrestricted grant by TEVA Germany, purveyors of glatiramer acetate. The unrestricted grant was spent for the production of the study material, distribution, compensation of the subinvestigator, collecting the data by a clinical research associate and statistical analysis. TZ and SK received no financial compensation for their role in the study and manuscript preparation.

\section{References}

I. Krupp LB, Pollina DA: Mechanisms and management of fatigue in progressive neurological disorders. Current opinion in neurology 1996, 9(6):456-460.

2. Fisk JD, Pontefract A, Ritvo PG, Archibald CJ, Murray TJ: The impact of fatigue on patients with multiple sclerosis. The Canadian journal of neurological sciences 1994, 21 (1):9-14.
3. Freal JE, Kraft GH, Coryell JK: Symptomatic fatigue in multiple sclerosis. Arch Phys Med Rehabil 1984, 65(3):135-138.

4. Vercoulen JH, Hommes OR, Swanink CM, Jongen PJ, Fennis JF, Galama JM, Meer JW van der, Bleijenberg G: The measurement of fatigue in patients with multiple sclerosis. A multidimensional comparison with patients with chronic fatigue syndrome and healthy subjects. Archives of neurology 1996, 53(7):642-649.

5. Schwid SR, Covington M, Segal BM, Goodman AD: Fatigue in multiple sclerosis: current understanding and future directions. J Rehabil Res Dev 2002, 39(2):2I I-224.

6. Amato MP, Ponziani G, Rossi F, Lied CL, Stefanile C, Rossi L: Quality of life in multiple sclerosis: the impact of depression, fatigue and disability. Multiple sclerosis (Houndmills, Basingstoke, England) 200I, 7(5):340-344.

7. Janardhan V, Bakshi R: Quality of life in patients with multiple sclerosis: the impact of fatigue and depression. Journal of the neurological sciences 2002, 205(I):5I-58.

8. Lobentanz IS, Asenbaum S, Vass K, Sauter C, Klosch G, Kollegger H, Kristoferitsch W, Zeitlhofer J: Factors influencing quality of life in multiple sclerosis patients: disability, depressive mood, fatigue and sleep quality. Acta neurologica Scandinavica 2004, I I0(I):6-13.

9. Flachenecker P, Kumpfel T, Kallmann B, Gottschalk M, Grauer O, Rieckmann P, Trenkwalder C, Toyka KV: Fatigue in multiple sclerosis: a comparison of different rating scales and correlation to clinical parameters. Multiple sclerosis (Houndmills, Basingstoke, England) 2002, 8(6):523-526.

10. Tellez N, Rio J, Tintore M, Nos C, Galan I, Montalban X: Does the Modified Fatigue Impact Scale offer a more comprehensive assessment of fatigue in MS? Multiple sclerosis (Houndmills, Basingstoke, England) 2005, I I (2): I 98-202.

II. Jackson MF, Quaal C, Reeves MA: Effects of multiple sclerosis on occupational and career patterns. Axone (Dartmouth, NS) I99I, I3(I):16-17. 20-12

12. Smith MM, Arnett PA: Factors related to employment status changes in individuals with multiple sclerosis. Multiple sclerosis (Houndmills, Basingstoke, England) 2005, I I (5):602-609.

13. Comi G, Leocani L, Rossi P, Colombo B: Physiopathology and treatment of fatigue in multiple sclerosis. Journal of neurology 200I, 248(3): 174-179.

14. Krupp LB: Fatigue in multiple sclerosis: definition, pathophysiology and treatment. CNS drugs 2003, I7(4):225-234.

15. Kesselring J, Thompson AJ: Spasticity, ataxia and fatigue in multiple sclerosis. Bailliere's clinical neurology 1997, 6(3):429-445.

16. Kern S, Ziemssen T: Brain immune communication psychoneuroimmunology of multiple sclerosis. Mult Scler 2007.

17. Zifko UA: Management of fatigue in patients with multiple sclerosis. Drugs 2004, 64( (12):1295-1304.

18. Pucci E, Branas P, D'Amico R, Giuliani G, Solari A, Taus C: Amantadine for fatigue in multiple sclerosis. Cochrane database of systematic reviews (Online) 2007:CD0028I8.

19. Rammohan KW, Rosenberg JH, Lynn DJ, Blumenfeld AM, Pollak CP, Nagaraja HN: Efficacy and safety of modafinil (Provigil) for the treatment of fatigue in multiple sclerosis: a two centre phase 2 study. Journal of neurology, neurosurgery, and psychiatry 2002, 72(2): 179-183. 
20. Stankoff B, Waubant E, Confavreux C, Edan G, Debouverie M, Rumbach L, Moreau T, Pelletier J, Lubetzki C, Clanet M: Modafinil for fatigue in MS: a randomized placebo-controlled double-blind study. Neurology 2005, 64(7): | |39- I | 43.

21. Corboy JR, Goodin DS, Frohman EM: Disease-modifying Therapies for Multiple Sclerosis. Current treatment options in neurology 2003, 5(I):35-54.

22. Metz LM, Patten SB, Archibald CJ, Bakker JI, Harris CJ, Patry DG, Bell RB, Yeung M, Murphy WF, Stoian CA, et al:: The effect of immunomodulatory treatment on multiple sclerosis fatigue. Journal of neurology, neurosurgery, and psychiatry 2004, 75(7): 1045-1047.

23. McDonald WI, Compston A, Edan G, Goodkin D, Hartung HP, Lublin FD, McFarland HF, Paty DW, Polman CH, Reingold SC, et al.: Recommended diagnostic criteria for multiple sclerosis: guidelines from the International Panel on the diagnosis of multiple sclerosis. Annals of neurology 200I, 50(I):I2I-I27.

24. Kurtzke JF: Rating neurologic impairment in multiple sclerosis: an expanded disability status scale (EDSS). Neurology 1983, 33(II): I 1444-1452.

25. Multiple Sclerosis Council for Clinical Practice Guidelines: Fatigue and multiple sclerosis: evidence-based management strategies for fatigue in multiple sclerosis. Washington DC: Paralyzed Veterans of America; 1998: I-33.

26. Heesen C, Nawrath L, Reich C, Bauer N, Schulz KH, Gold SM Fatigue in multiple sclerosis: an example of cytokine mediated sickness behaviour? Journal of neurology, neurosurgery, and psychiatry 2006, 77(I):34-39.

27. Flachenecker P, Bihler I, Weber F, Gottschalk M, Toyka KV, Rieckmann P: Cytokine mRNA expression in patients with multiple sclerosis and fatigue. Multiple sclerosis (Houndmills, Basingstoke, England) 2004, 10(2):165-169.

28. Ziemssen T, Kumpfel T, Klinkert WE, Neuhaus O, Hohlfeld R: Glatiramer acetate-specific $\mathrm{T}$-helper I- and 2-type cell lines produce BDNF: implications for multiple sclerosis therapy. Brain-derived neurotrophic factor. Brain 2002, I25(Pt II):238I-239I.

29. Ziemssen T, Reichmann H: Non-motor dysfunction in Parkinson's disease. Parkinsonism \& related disorders 2007, I3(6):323-332.

30. Tartaglia MC, Narayanan S, Francis SJ, Santos AC, De Stefano N, Lapierre $\mathrm{Y}$, Arnold DL: The relationship between diffuse axonal damage and fatigue in multiple sclerosis. Archives of neurology 2004, 6 I(2):20I-207.

31. Tedeschi G, Dinacci D, Lavorgna L, Prinster A, Savettieri G, Quattrone A, Livrea P, Messina C, Reggio A, Servillo G, et al.: Correlation between fatigue and brain atrophy and lesion load in multiple sclerosis patients independent of disability. Journal of the neurological sciences 2007, 263(I-2): 15-19.

32. Rocca MA, Agosta F, Colombo B, Mezzapesa DM, Falini A, Comi G, Filippi M: fMRI changes in relapsing-remitting multiple sclerosis patients complaining of fatigue after IFNbeta-la injection. Human brain mapping 2007, 28(5):373-382.

33. Khan O, Mackenzie M, Shen Y, Zak I, Latif Z, Caon C: Combined Brain MTR and H-MRS Multi-Modality Approach To Investigate Mechanism of Action of Interferon Beta and Glatiramer Acetate in RRMS. Neurology 2007.

34. Khan O, Shen Y, Bao F, Caon C, Tselis A, Latif Z, Zak I: Long-Term Study of Brain (I)H-MRS Study in Multiple Sclerosis: Effect of Glatiramer Acetate Therapy on Axonal Metabolic Function and Feasibility of Long-Term (I)H-MRS Monitoring in Multiple Sclerosis. J Neuroimaging 2007.

35. Lage MJ, Castelli-Haley J, Oleen-Burkey MA: Effect of immunomodulatory therapy and other factors on employment loss time in multiple sclerosis. Work (Reading, Mass) 2006 27(2):|43-|5|.

36. Ziemssen T, Neuhaus $O$, Hohlfeld R: Risk-benefit assessment of glatiramer acetate in multiple sclerosis. Drug Saf 200I, 24(13):979-990.

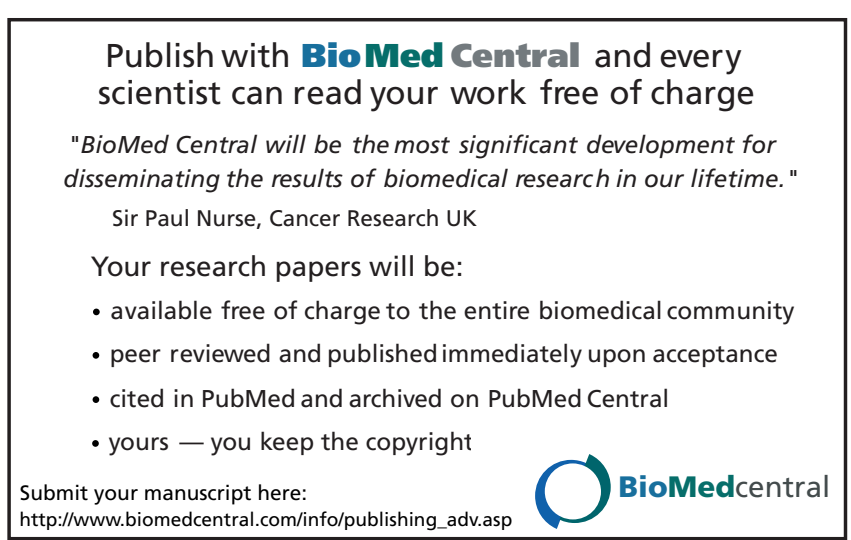

